\title{
CTCs: a prognostic marker for prostate cancer
}

Circulating tumor cell (CTC) numbers before and during first-line chemotherapy predict survival and can be used to monitor response to treatment in patients with progressive, metastatic, castration-resistant prostate cancer. Scher et al. report that CTC count is a stronger prognostic biomarker than prostate-specific antigen (PSA).

The researchers retrospectively analyzed data from the IMMC38 trial, which showed that a CTC count greater than 5 per $7.5 \mathrm{ml}$ of blood before and after treatment predicted poor overall survival. Data from 164 men with progressive, metastatic, castration-resistant prostate cancer scheduled to undergo first-line chemotherapy were assessed in the present analysis, by which time 103 patients had died (median survival 18.5 months).

Scher's team found that at baseline (pretreatment), elevated CTC counts, PSA titers, and lactate dehydrogenase concentrations were all associated with a high risk of death. CTC and PSA levels were also measured before each chemotherapy cycle; change in CTC count relative to baseline at 4,8 , and 12 weeks after treatment was a strong indicator of the risk for death, whereas the change in PSA titer was only weakly associated with survival. The investigators noted that outcome was best predicted by lactate dehydrogenase concentration and CTC counts at baseline, and CTC counts after treatment.

Biomarkers such as CTC counts could be used as an intermediate end point of survival, potentially shortening the time line for drug approval.

\section{Lisa Richards}

Original article Scher, H. I. et al. Circulating tumour cells as prognostic markers in progressive, castration-resistant prostate cancer: a reanalysis of IMMC38 trial data. Lancet Oncol. 10, 233-239 (2009). 